\title{
Self-organization and shape change by active polarization in nematic droplets
}

\author{
Fabian Jan Schwarzendahl $\odot,{ }^{1,2}$ Pierre Ronceray $\odot,{ }^{3}$ Kimberly L. Weirich $\odot,{ }^{4}$ and Kinjal Dasbiswas $\odot$ 1,* \\ ${ }^{1}$ Department of Physics, University of California Merced, Merced, California 95343, USA \\ ${ }^{2}$ Institut für Theoretische Physik II: Weiche Materie, Heinrich-Heine-Universität Düsseldorf, 40225 Düsseldorf, Germany \\ ${ }^{3}$ Aix Marseille Univ, Université de Toulon, CNRS, CINAM, Turing Center for Living Systems, Marseille, France \\ ${ }^{4}$ Department of Materials Science and Engineering, Clemson University, Clemson, South Carolina 29634, USA
}

(Received 11 March 2021; revised 30 July 2021; accepted 29 September 2021; published 22 October 2021)

\begin{abstract}
Active forces drive critical biological processes such as spontaneous organization and shape change during cell division. Here, we present a minimal hydrodynamic model leading to a unified description of self-organization and division in nematic droplets through active polarity sorting of cytoskeletal filaments by molecular motors. We find that motors self-organize within droplets while structuring filaments into polarized aster defects. At large activity, motors deform droplets leading to multidroplet chains and droplet division, consistent with experiments on actomyosin tactoids. We predict droplet steady-state phase diagrams that inform programmable shape changes in confined soft materials.
\end{abstract}

DOI: 10.1103/PhysRevResearch.3.043061

\section{INTRODUCTION}

Active mechanical forces enable biological cells, to move, change shape, organize components, and divide. Subcellular cytoskeletal assemblies, comprising polar filaments (such as actin) and molecular motors (such as myosin) that transduce biochemical reactions to generate active mechanical forces, drive these processes [1]. Understanding the general physical principles of living matter provides insight into cell biology, and also guides the engineering of artificial cells and systems that exhibit spatiotemporal organization of components and spontaneous shape change [2]. Experiments on cytoskeletal extracts capture elements of cell biological phenomena with only a fraction of the biochemical complexity occurring in vivo, and exhibit a rich array of collective phenomena [3-5] described by active matter theory [6].

Spatiotemporal organization in cells can be achieved through phase separation into droplets which typically lack order and activity [7]. In contrast, cytoskeletal filaments can form phase-separated droplets with orientational order [8]. This nematic order confers an equilibrium spindle shape to these droplets [9], known as tactoids, which arises from a competition of droplet surface tension, filament alignment at the interface, and nematic elasticity $[10,11]$. When confined to a droplet, active forces can drive nonequilibrium biomimetic phenomena such as droplet shape change [12-16], and motility $[17,18]$ with dynamics governed by the geometry of the confining droplet [19]. Cytoskeletal systems can form active

\footnotetext{
*kdasbiswas@ucmerced.edu

Published by the American Physical Society under the terms of the Creative Commons Attribution 4.0 International license. Further distribution of this work must maintain attribution to the author(s) and the published article's title, journal citation, and DOI.
}

nematic states characterized by large-scale flows and chaotic dynamics, but these can be transient since motors eventually cluster causing localized stresses [20]. At steady state, the directed "walking" of motors on filaments, along with motorbased filament crosslinking, can lead to polar order, where filaments point in the same direction in local regions. Such polar active states, including their defect structures such as asters and vortices [21], are fruitfully described by hydrodynamic theories for bulk systems [22-28].

In recent experiments, myosin motors were shown to selforganize at the midplane of actin-based nematic droplets [29]. When sufficiently active, motors deform the droplet, even splitting it into two. A simple free energy-based model considering only the mutual alignment of the filaments and motor clusters was invoked to capture these key behaviors, but relied on arguments specific to the shape and arrangement of the droplet components [8]. To develop a more general mechanism, we now consider if these dynamics can be driven by active mechanical forces, specifically the directed sliding of filaments by motors leading to their sorting by polarity [1].

In this paper, we combine continuum modeling for the phase separation of equilibrium nematic droplets with an active polar model describing how motors move and slide filaments. Using numerical simulations, complemented by theoretical analysis, we show that the resulting motor-filament self-organization destabilizes droplets. This gives rise to a rich array of experimentally observed shapes including deformed, divided, and multidroplet chains that can be generated through tuning motor activity.

\section{ONE-DIMENSIONAL POLARITY SORTING MODEL}

We first examine a simplified one-dimensional (1D) setup of motors interacting with filaments, as sketched in Fig. 1(a). Here, red lines depict actin filaments, blue circles show myosin II motors (which occur in experiments as polymerized 




FIG. 1. Illustration of steps in the active polarity-sorting model. (a) Motors "walk" towards barbed ends of filaments which they slide in the opposite direction as a result of momentum conservation. (b) Polarization and (c) motor density at steady state as a function of position in a one-dimensional model that shows motor localization to center of the bundle by polarity sorting. (d) In the 2D nematic droplet model, the filaments are initially oriented along the long axis without any polarity preference. Motors bind to filaments and advect them according to their polarity. (e) At steady state, motors gather at the droplet midplane and sort filaments into an aster that deforms the droplet..

minifilaments [30]), while black arrows indicate the direction of motion. This scenario arises in contractile actin bundles $[31,32]$ and along the local director in a two-dimensional (2D) nematic. Since motors walk towards specific ends of the polar filament, we consider a density of left and right pointing filaments, denoted by $n_{+}$and $n_{-}$, respectively. Momentum balance for the motor-filament system requires that a right (left) pointing filament is pushed to the right (left) by the motor as the motor moves in the other direction along the polar filament. The active motion of motors of density $m$ thus gives rise to fluxes of right and left pointing filaments that can be expressed as $J_{+}=\zeta n_{+} m$ and $J_{-}=-\zeta n_{-} m$, respectively. $\zeta$ is proportional to the sliding velocity of filaments. The net flux of motors is written as $-v_{0}\left(n_{+}-n_{-}\right) m$, where $v_{0}$ is the self-propulsion velocity of motors and $v_{0} / \zeta$ depends on the relative drag, and therefore sizes, of the motor minifilaments and actin filaments.

Including the diffusion of filaments and motors with coefficients $D$ and $D_{m}$, respectively, the flux conservation equations in terms of filament density $\rho=n_{+}+n_{-}$and the 1D polarization $p=\left(n_{+}-n_{-}\right) / \rho_{0}$ of filaments, where $\rho_{0}=\langle\rho\rangle$ is the average density, gives

$$
\begin{aligned}
& \partial_{t} \rho=D \partial_{x}^{2} \rho-\zeta \rho_{0} \partial_{x}(p m), \\
& \partial_{t} p=D \partial_{x}^{2} p-\zeta / \rho_{0} \partial_{x}(\rho m), \\
& \partial_{t} m=D_{m} \partial_{x}^{2} m+v_{0} \partial_{x}(m p) .
\end{aligned}
$$

The steady-state solution of Eqs. (2) and (3) [see Supplemental Material (SM) [33] Sec. S1], is the one-dimensional equivalent of an aster, where the populations of right (left) pointing filaments are completely sorted to the right (left) of the aster, and is shown in Figs. 1(b) and 1(c). In this minimal model, we do not yet consider the role of myosin motors as active crosslinkers which is the main source of filament flux in related systems [34]. This makes the proposed mechanism for motor movements clearer and is justified in our experimental system since the myosin minifilaments are large and can drive individual short actin filaments (see SM Sec. S1). We do include the effect of motor-based crosslinking phenomenologically in the 2D hydrodynamic model we present next. The simple 1D model reproduces the key experimental observation that motors migrate to the center of the droplet, and predicts that they induce strong polarity sorting.

\section{TWO-DIMENSIONAL NEMATIC DROPLET WITH MOTOR-INDUCED POLARIZATION}

To explore this prediction of active polarity sorting in nematic droplets and its implications for the droplet shape, we build a more realistic hydrodynamic description for a suspension of filaments and motors. In contrast with a thin fluid film, the filaments here aggregate into droplets with free interfaces that separate the high-density nematic from the low-density isotropic phases, depicted in Fig. 1(d). This is conveniently described by a nondimensional "phase field" corresponding to filament density $\psi$, where $\psi>0(\psi<0)$ describes the interior (exterior) of the droplet. Filaments in the high-density droplet interior align in orientation, described by the $2 \mathrm{D}$ nematic order parameter $Q_{i j}$. These two ingredients result in nematic droplets with a tactoid shape at equilibrium [35]. The active motion of filaments and polarity sorting induced by motors results in a net polarization within the droplet. Generalizing Eqs. (1)-(3) to 2D, and observing the usual principles of conservation and symmetry [6], we obtain the dynamical equations (see SM Sec. S2),

$$
\begin{aligned}
& \partial_{t} m=D_{m} \nabla^{2} m+v_{0} \nabla \cdot(m \boldsymbol{p})+k_{\mathrm{on}} \psi \theta(\psi)-k_{\mathrm{off}} m \\
& \partial_{t} \psi=\Gamma_{\psi} \nabla^{2} \frac{\delta F}{\delta \psi}-\zeta \nabla \cdot(m \boldsymbol{p}) \\
& \partial_{t} \boldsymbol{p}=-\zeta_{0} \nabla \psi-\zeta_{p} \nabla m-\Gamma_{p} \frac{\delta F}{\delta \boldsymbol{p}} \\
& \partial_{t} Q_{i j}=-\Gamma_{Q} \frac{\delta F}{\delta Q_{i j}}
\end{aligned}
$$

Equations (4) and (5) describe the conservation of motors and filaments, respectively, and include both active and passive fluxes on the right side. The motor flux includes diffusion in the first term, and active motion with a propulsion velocity $v_{0}$ in the second. Additionally, we include bindingunbinding kinetics for the motors [third and fourth terms in Eq. (4)], where motors bind with rate $k_{\text {on }}$ wherever filaments exist [expressed by $\theta(\psi)$, the Heaviside step function] from a "reservoir" of free motors in the solution, and unbind with rate $k_{\text {off }}$, but are not restricted to diffuse within the droplet. Equation (5) includes a flux created by a free energy $F$ of interfilament interactions [full form given below in Eq. (8)] as well as an active flux induced by motors advecting filaments along their polarity. Equation (6) describes the induction of polarization by torques caused by gradients in motor and filament density, and its relaxation through rotational diffusion. The $\Gamma$ coefficients represent inverse dissipative timescales. In the framework of the Toner-Tu theory of polar active fluids [36], where $\boldsymbol{p}$ is both an orientational order parameter 
and a fluid velocity, the $-\zeta_{0} \nabla \psi$ term gives the gradient of pressure, and $-\zeta_{p} \nabla m$ is the gradient of an active stress created by motors [37]. Similar terms arise in the theory of chemotactic colloids [38] and equilibrium polar liquid crystals [39], where a chemical concentration can guide polarization. Microscopically, the $\zeta_{p}$ term captures the preferred orientation of filaments towards motor-rich regions via the binding and crosslinking by motors, while $\zeta$ originates from the sliding of individual filaments by motors. Note that the $\zeta$ specifies both polarization and active flux in the one-dimensional model, whereas the $\zeta_{p}$ term arises in the two-dimensional model because of the additional orientational degree of freedom. The parameters $v_{0}, \zeta$, and $\zeta_{p}$ then all arise from motor activity but depend differently on the size and crosslinking properties of the motor cluster. In Eq. (7), the equilibrium interactions dominate over any realignment of the nematic by fluid flows which are damped out.

The equilibrium dynamics assume a coupled phase transition in $\psi$ and nematic order $\boldsymbol{Q}$, and a relaxation of $\boldsymbol{p}$. These are minimally described by a total free energy adapted from a coarse-grained phase field model for lyotropic liquid crystals [40],

$$
\begin{aligned}
F & =\int d^{2} \mathbf{x}\left(f_{\psi}+f_{p}+f_{Q}+f_{\text {int }}\right), \\
f_{\psi} & =-\frac{\nu_{2}}{2} \psi^{2}+\frac{\nu_{4}}{4} \psi^{4}+\frac{B}{2}(\nabla \psi)^{2}, \\
f_{p} & =\frac{\alpha_{p}}{2}|\boldsymbol{p}|^{2}+\frac{\beta_{p}}{2}|\boldsymbol{p}|^{4}+\frac{\kappa_{p}}{2}(\nabla \cdot \boldsymbol{p})^{2}, \\
f_{Q} & =\frac{\alpha_{Q}}{4} Q_{i j}^{2}+\frac{\beta_{Q}}{16} Q_{i j}^{2} Q_{k h}^{2}+\frac{\kappa_{Q}}{2}\left(\partial_{k} Q_{i j}\right)^{2}, \\
f_{\text {int }} & =\frac{C}{4} Q_{i j}^{2} \psi(\psi-1)+A \partial_{i} \psi \partial_{j} Q_{i j}-\Omega Q_{i j} p_{i} p_{j} .
\end{aligned}
$$

Equation (8b) gives the standard Cahn-Hilliard model for phase-separating droplets. Ignoring corrections for curved interfaces [41], the droplet surface tension ("line tension" in 2D) is $\gamma=\frac{2 \sqrt{2 B v_{2}^{3}}}{3 v_{4}}$ [42]. Equation (8c) includes two relaxation terms, $\alpha_{p}, \beta_{p}>0$ (corresponding to a lack of spontaneous polar order) and an elasticity $\kappa_{p}$. Equation (8d) is the Landau-de Gennes free energy for the nematic order $Q_{i j}$ with elasticity $\kappa_{Q}$. The equilibrium couplings between fields are written in Eq. (8e). The three terms correspond to, respectively, the density-driven isotropic-nematic transition that induces nematic order within the droplet, a "weak anchoring" that aligns the nematic parallel to the droplet interface $(A>0)$, and an alignment of the polarization with the nematic order $(\Omega>0)$. Overall, this model recapitulates the key elements of nematic phase separation of the filaments and their coupling with motor activity.

\section{RESULTS}

We now employ numerical simulations to explore the consequences of motor activity on the dynamics and morphology of phase-separating nematic droplets. Starting from an initially circular droplet of radius $R_{0}$, we integrate Eqs. (4)-(7) until a nonequilibrium steady state is reached (see SM Sec. S2). Figure 2 presents typical simulation results. Our
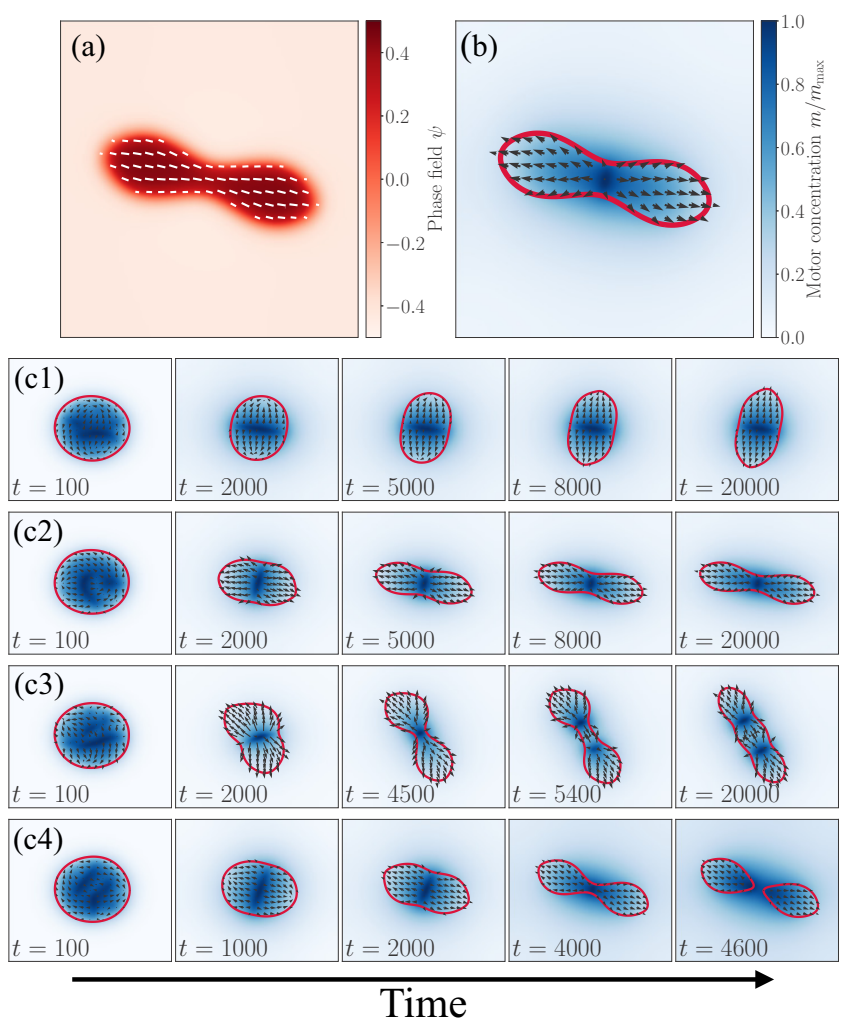

FIG. 2. (a) and (b) A representative numerical steady-state solution of our active droplet model. (a) The red gradient indicates the density $\psi$ and the white lines indicate the nematic director field. (b) The blue gradient, black arrows, and the red line indicate the motor concentration, the polarization direction, and the droplet boundary, respectively. (c) Time series for different droplet types observed in our simulations: (c1) single tactoid with centering aster; (c2) two connected tactoids with centering aster; (c3) three connected tactoids with two asters; (c4) fully divided droplet. The number in each snapshot shows the simulation time.

model is a general description of the active motor-induced forces within a droplet of cytoskeletal filaments and is not meant to capture all the details of equilibrium shape of the three-dimensional (3D) experimental actomyosin tactoids of Ref. [29]. Model parameter choices are then made (see SM Tables S2 and S3) to get the characteristic tactoid shapes as a proof of concept. We obtain an elongated droplet with the nematic aligned along its long axis, as shown by the density and nematic director plots for a typical case in Fig. 2(a). The motor density accumulates at the core of the aster and induces an outward polarization as shown in Fig. 2(b). We first explore the interplay between motor-generated active forces, which tend to distort the equilibrium structure, and surface tension which resists such deformation. To this aim, we perform simulations with varying motor-induced polarization $\zeta_{p}$ relative to the surface tension $\gamma$. The resulting time sequences of the observed droplet shapes, starting from an unpolarized droplet, are shown in Fig. 2(c). When $\zeta_{p}$ is low compared to surface tension, Fig. 2(c1), motors localize towards the center to form an aster, which only slightly polarizes and deforms the droplet. At intermediate $\zeta_{p}$ [Fig. 2(c2)], motors localize more strongly, resulting in a stronger elongation and the appearance 

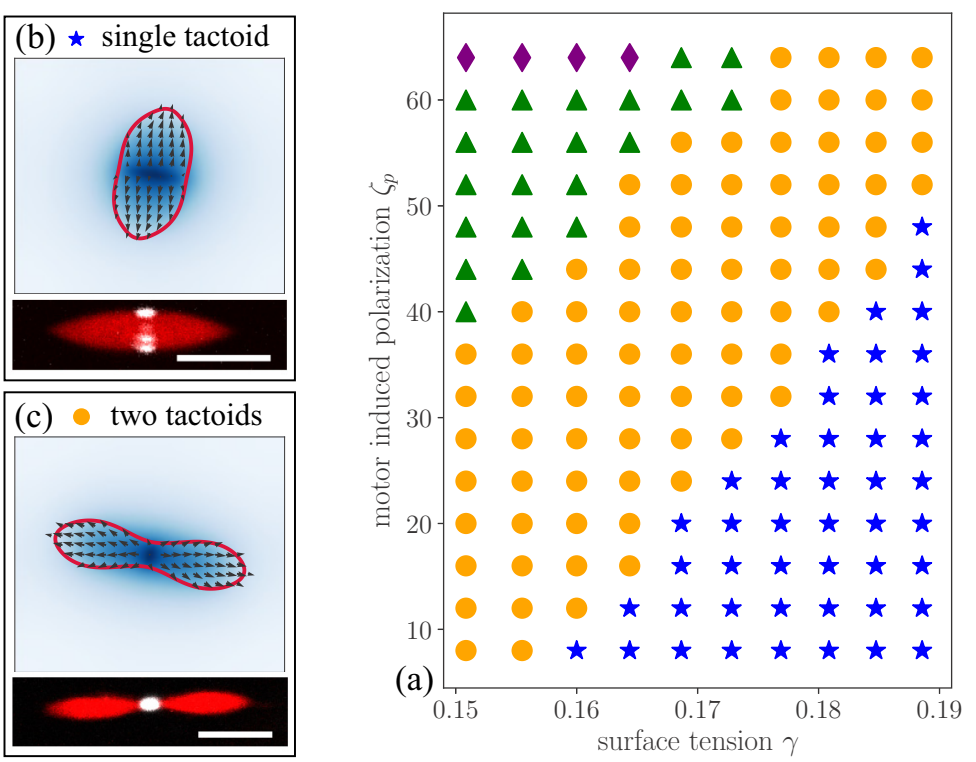
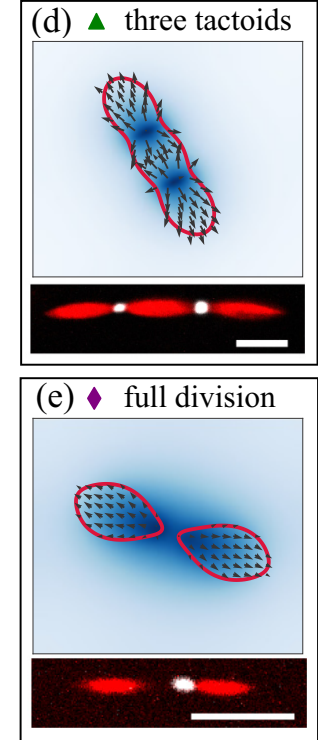
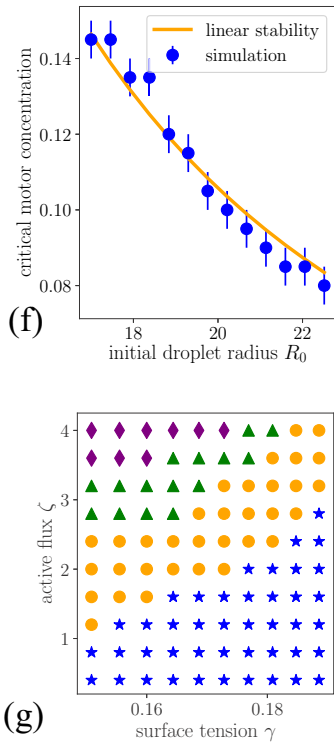

FIG. 3. (a) Nonequilibrium phase diagram from our simulations for varying surface tension $\gamma$ and active stress $\zeta_{p}$ while keeping $\zeta=1.5$ fixed. (b)-(e) Different steady-state shapes observed in our simulations and related experimental images (see SM Sec. S3 and Ref. [29] for details) of actomyosin tactoids (myosin motors in white, actin in red, scale bars $5 \mu \mathrm{m}$ ). For the simulation results (b)-(e) (top) the color code is the motor concentration $c$, black arrows are the polarization $\boldsymbol{p}$, and the red contour shows the shape of the droplet. We classify the droplet shapes as follows: (b) single tactoid with a centering aster; (c) two tactoids with one aster; (d) three connected tactoids with two asters; (e) fully divided droplet. (f) Critical motor concentration for the transition from one to two asters as a function of initial droplet size. (g) Nonequilibrium phase diagram showing the different steady states observed in our simulations for varying active flux $\zeta$ and surface tension $\gamma$ with $\zeta_{p}=12.5$ fixed.

of a constriction of the droplet around the midplane between strongly polarized lobes. Increasing the $\zeta_{p}$ further can lead to two distinct scenarios: In Fig. 2(c3), the aster divides and a third, central lobe with strong polarity gradient emerges between two constrictions. In Fig. 2(c4), finally, $\zeta_{p}$ is sufficiently strong to induce full division of the initial droplet into two polarized daughter droplets. The short-time dynamics of this model thus reproduces the motor centering, aster formation, and polarity sorting features of the 1D model (Fig. 1), while at longer times a complex diversity of morphologies emerges from the interplay between surface tension and motor activity.

To rationalize this rich phenomenology, we build a morphological phase diagram in Fig. 3(a) in $\zeta_{p}-\gamma$ while keeping other parameters constant. Each distinct steady-state structure is shown in Figs. 3(b)-3(e) (top). Interestingly, each of these morphologies corresponds to experimentally observed shapes in actomyosin droplets (see SM Sec. S3), as shown in Figs. 3(b)-3(e) (bottom). Confirming the qualitative findings described in Fig. 2(c), at medium to high surface tension and low $\zeta_{p}$, we find motors form asters in the midplane of the undeformed droplet (blue stars), whereas higher $\zeta_{p}$ increases the influence of the centered aster on droplet shape, deforming it into two lobes (orange circles). This is consistent with the experimental observation that motors always localize to a droplet midplane, but only deform the droplet when there are more active motors [29]. Qualitatively, the motors at the aster core splay the filaments, which are anchored to the interface, ultimately resulting in an inward deformation of the interface. By balancing the effective free energy of motor-induced splay [43] against surface tension (see SM Sec. S4), we obtain a criterion for droplet deformation, $\zeta_{p} \sim \gamma$, consistent with the phase boundary in Fig. 3(a).

By further increasing $\zeta_{p}$ and lowering surface tension, we find droplets with two asters which deform them into three connected tactoids [Fig. 3(d), green triangles]. Importantly, we also find experimental realizations of such multiply deformed, equilobed structures, shown in Fig. 3(d), bottom panel. Such "strings of tactoids" connected by multiple motor clusters are evocative of fibers with periodic contractile units such as in muscle or anomalous, multipolar biological spindles [44].

Multiaster states such as aster lattices are a generic feature of bulk active polar fluids [24,28], but here, we analyze their occurrence within droplets. To explore the aster-forming instability arising from the feedback between motor flux and motor-induced polarization in Eqs. (4) and (6), we perform a linear stability analysis to identify a characteristic aster spacing (see SM Sec. S5). We find in Fig. 3(f) that the critical motor concentration for transition from one to two asters decreases with initial droplet size, consistent with a predicted scaling of $m_{0} \sim 1 / l_{d}^{2}$. At even higher $\zeta_{p}$ and lower $\gamma$, we find a region of the phase diagram in Fig. 3(a) where the droplet is fully divided (purple diamonds). We also find that the phase diagram is sensitive to droplet size, larger droplets being more unstable (see SM Sec. S7).

To see how droplet division can be enhanced, we now explore another shape instability in our model Eq. (5), whereby an active density flux $\zeta$ pushes filaments away from the aster. By varying $\zeta$ and $\gamma$, while keeping $\zeta_{p}(=12.5)$ fixed, we obtain the phase diagram in Fig. $3(\mathrm{~g})$, which shows undeformed 
single droplets (blue stars), deformed droplets (orange circles), three connected droplets (green triangles), and fully divided droplets (purple diamonds). The effect of the filament flux on droplet shape can be captured by an effective freeenergy argument (see SM Sec. S6), yielding a criterion for division $(\zeta \sim \gamma)$ consistent with simulations.

\section{CONCLUSIONS}

We build a minimal theoretical model for motor-filament droplets which captures four different shapes, and show them experimentally in the actomyosin tactoids. We predict a phase diagram of droplet shapes based on motor activity and droplet mechanical energy, and find that larger droplets are more unstable, which indicates a size selection. While motor activity and droplet size have not been systematically varied in current experiments, the model provides predicted dependencies that can be tested in future experiments. Unlike other theoretically proposed active droplet division mechanisms, the shape changes we predict do not require fluxes of chemical reactants [13], fluid flows and defect dynamics [12], or chaotic instabilities [45] that arise in active nematic morphodynamics [46]. Instead, we find the emergence of local polar order within a nematic and the resulting spatial localization of activity crucial to capture the shape changes. In addition to in vitro cytoskeletal spindles, our results also have implications for the physical principles behind cell division and the organization of the biological spindle [47-49]. We expect our work to inform strategies to use the localization of active agents to achieve programmable shape changes in materials [50] and synthetic cells.

\section{ACKNOWLEDGMENTS}

F.J.S and K.D. gratefully acknowledge computing time on the Multi-Environment Computer for Exploration and Discovery (MERCED) cluster (NSF Grant No. ACI-1429783) and support from the NSF-CREST: Center for Cellular and Biomolecular Machines (CCBM) at the University of California, Merced (NSF-HRD-1547848). P.R. received funding from the "Investissements d'Avenir" French Government program managed by the French National Research Agency (ANR-16-CONV-0001) and from Excellence Initiative of Aix-Marseille University - A*MIDEX. We thank C. C. Maass and M. G. Mazza for a critical reading of the manuscript and K. Husain for useful discussions. K.D. benefited from workshops organized by the Kavli Institute of Theoretical Physics with support from the National Science Foundation under Grant No. NSF PHY-1748958.
[1] M. Murrell, P. W. Oakes, M. Lenz, and M. L. Gardel, Forcing cells into shape: the mechanics of actomyosin contractility, Nat. Rev. Mol. Cell Biol. 16, 486 (2015).

[2] V. Noireaux, Y. T. Maeda, and A. Libchaber, Development of an artificial cell, from self-organization to computation and self-reproduction, Proc. Natl. Acad. Sci. USA 108, 3473 (2011).

[3] M. Soares e Silva, M. Depken, B. Stuhrmann, M. Korsten, F. C. MacKintosh, and G. H. Koenderink, Active multistage coarsening of actin networks driven by myosin motors, Proc. Natl. Acad. Sci. USA 108, 9408 (2011).

[4] T. Sanchez, D. T. Chen, S. J. DeCamp, M. Heymann, and Z. Dogic, Spontaneous motion in hierarchically assembled active matter, Nature (London) 491, 431 (2012).

[5] L. Huber, R. Suzuki, T. Krüger, E. Frey, and A. R. Bausch, Emergence of coexisting ordered states in active matter systems, Science 361, 255 (2018).

[6] M. C. Marchetti, J. F. Joanny, S. Ramaswamy, T. B. Liverpool, J. Prost, M. Rao, and R. A. Simha, Hydrodynamics of soft active matter, Rev. Mod. Phys. 85, 1143 (2013).

[7] A. A. Hyman, C. A. Weber, and F. Jülicher, Liquid-liquid phase separation in biology, Annu. Rev. Cell Dev. Biol. 30, 39 (2014).

[8] K. L. Weirich, S. Banerjee, K. Dasbiswas, T. A. Witten, S. Vaikuntanathan, and M. L. Gardel, Liquid behavior of crosslinked actin bundles, Proc. Natl. Acad. Sci. USA 114, 2131 (2017).

[9] J. Brugués and D. Needleman, Physical basis of spindle selforganization, Proc. Natl. Acad. Sci. USA 111, 18496 (2014).

[10] A. V. Kaznacheev, M. M. Bogdanov, and S. A. Taraskin, The nature of prolate shape of tactoids in lyotropic inorganic liquid crystals, J. Exp. Theor. Phys. 95, 57 (2002).
[11] P. Prinsen and P. van der Schoot, Shape and director-field transformation of tactoids, Phys. Rev. E 68, 021701 (2003).

[12] L. Giomi and A. DeSimone, Spontaneous Division and Motility in Active Nematic Droplets, Phys. Rev. Lett. 112, 147802 (2014).

[13] D. Zwicker, R. Seyboldt, C. A. Weber, A. A. Hyman, and F. Jülicher, Growth and division of active droplets provides a model for protocells, Nat. Phys. 13, 408 (2017).

[14] C. A. Whitfield and R. J. Hawkins, Instabilities, motion and deformation of active fluid droplets, New J. Phys. 18, 123016 (2016).

[15] R. Singh and M. E. Cates, Hydrodynamically Interrupted Droplet Growth in Scalar Active Matter, Phys. Rev. Lett. 123, 148005 (2019).

[16] H. R. Vutukuri, M. Hoore, C. Abaurrea-Velasco, L. van Buren, A. Dutto, T. Auth, D. A. Fedosov, G. Gompper, and J. Vermant, Active particles induce large shape deformations in giant lipid vesicles, Nature (London) 586, 52 (2020)

[17] E. Tjhung, D. Marenduzzo, and M. E. Cates, Spontaneous symmetry breaking in active droplets provides a generic route to motility, Proc. Natl. Acad. Sci. USA 109, 12381 (2012).

[18] F. Ziebert, S. Swaminathan, and I. S. Aranson, Model for selfpolarization and motility of keratocyte fragments, J. R. Soc., Interface 9, 1084 (2012).

[19] F. C. Keber, E. Loiseau, T. Sanchez, S. J. DeCamp, L. Giomi, M. J. Bowick, M. C. Marchetti, Z. Dogic, and A. R. Bausch, Topology and dynamics of active nematic vesicles, Science $\mathbf{3 4 5}$, 1135 (2014).

[20] N. Kumar, R. Zhang, J. J. de Pablo, and M. L. Gardel, Tunable structure and dynamics of active liquid crystals, Sci. Adv. 4, eaat7779 (2018) 
[21] T. Surrey, F. Nédélec, S. Leibler, and E. Karsenti, Physical properties determining self-organization of motors and microtubules, Science 292, 1167 (2001).

[22] H. Y. Lee and M. Kardar, Macroscopic equations for pattern formation in mixtures of microtubules and molecular motors, Phys. Rev. E 64, 056113 (2001).

[23] K. Kruse, J. F. Joanny, F. Jülicher, J. Prost, and K. Sekimoto, Asters, Vortices, and Rotating Spirals in Active Gels of Polar Filaments, Phys. Rev. Lett. 92, 078101 (2004).

[24] S. Sankararaman, G. I. Menon, and P. B. Sunil Kumar, Selforganized pattern formation in motor-microtubule mixtures, Phys. Rev. E 70, 031905 (2004).

[25] I. S. Aranson and L. S. Tsimring, Theory of self-assembly of microtubules and motors, Phys. Rev. E 74, 031915 (2006).

[26] A. Ahmadi, T. B. Liverpool, and M. C. Marchetti, Nematic and polar order in active filament solutions, Phys. Rev. E 72, 060901(R) (2005).

[27] F. Ziebert and W. Zimmermann, Nonlinear competition between asters and stripes in filament-motor systems, Eur Phys. J. E 18, 41 (2005).

[28] K. Gowrishankar and M. Rao, Nonequilibrium phase transitions, fluctuations and correlations in an active contractile polar fluid, Soft Matter 12, 2040 (2016).

[29] K. L. Weirich, K. Dasbiswas, T. A. Witten, S. Vaikuntanathan, and M. L. Gardel, Self-organizing motors divide active liquid droplets, Proc. Natl. Acad. Sci. USA 116, 11125 (2019).

[30] J. Howard, Mechanics of Motor Proteins and the Cytoskeleton (Sinauer Associates, Sunderland, MA, 2001).

[31] K. Kruse and F. Jülicher, Actively Contracting Bundles of Polar Filaments, Phys. Rev. Lett. 85, 1778 (2000).

[32] M. Stachowiak, P. McCall, T. Thoresen, H. Balcioglu, L. Kasiewicz, M. Gardel, and B. O'Shaughnessy, Selforganization of myosin ii in reconstituted actomyosin bundles, Biophys. J. 103, 1265 (2012).

[33] See Supplemental Material at http://link.aps.org/supplemental/ 10.1103/PhysRevResearch.3.043061 for additional explanations of the models and experiments.

[34] S. Fürthauer, B. Lemma, P. J. Foster, S. C. Ems-McClung, C.-H. Yu, C. E. Walczak, Z. Dogic, D. J. Needleman, and M. J. Shelley, Self-straining of actively crosslinked microtubule networks, Nat. Phys. 15, 1295 (2019).

[35] N. B. Ludwig, K. L. Weirch, E. Alster, T. A. Witten, M. L. Gardel, K. Dasbiswas, and S. Vaikuntanathan, Nucleation and shape dynamics of model nematic tactoids around adhesive colloids, J. Chem. Phys. 152, 084901 (2020).

[36] J. Toner and Y. Tu, Long-Range Order in a Two-Dimensional Dynamical XY Model: How Birds Fly Together, Phys. Rev. Lett. 75, 4326 (1995).

[37] K. Husain and M. Rao, Emergent Structures in an Active Polar Fluid: Dynamics of Shape, Scattering, and Merger, Phys. Rev. Lett. 118, 078104 (2017).

[38] B. Liebchen and H. Löwen, Synthetic chemotaxis and collective behavior in active matter, Acc. Chem. Res. 51, 2982 (2018).

[39] W. Kung, M. C. Marchetti, and K. Saunders, Hydrodynamics of polar liquid crystals, Phys. Rev. E 73, 031708 (2006).

[40] H. Löwen, A phase-field-crystal model for liquid crystals, J. Phys.: Condens. Matter 22, 364105 (2010).

[41] J. C. Everts, M. T. J. J. M. Punter, S. Samin, P. van der Schoot, and R. van Roij, A Landau-de Gennes theory for hard colloidal rods: Defects and tactoids, J. Chem. Phys. 144, 194901 (2016).

[42] S. Safran, Statistical Thermodynamics of Surfaces, Interfaces, and Membranes (CRC Press, Boca Raton, FL, 2018).

[43] D. Pettey and T. C. Lubensky, Stability of texture and shape of circular domains of Langmuir monolayers, Phys. Rev. E 59, 1834 (1999).

[44] N. J. Ganem, S. A. Godinho, and D. Pellman, A mechanism linking extra centrosomes to chromosomal instability, Nature (London) 460, 278 (2009).

[45] I. Maryshev, A. B. Goryachev, D. Marenduzzo, and A Morozov, Dry active turbulence in a model for microtubulemotor mixtures, Soft Matter 15, 6038 (2019).

[46] L. Metselaar, J. M. Yeomans, and A. Doostmohammadi, Topology and Morphology of Self-Deforming Active Shells, Phys. Rev. Lett. 123, 208001 (2019).

[47] M. Leoni, O. V. Manyuhina, M. J. Bowick, and M. C. Marchetti, Defect driven shapes in nematic droplets: analogies with cell division, Soft Matter 13, 1257 (2017).

[48] J. Roostalu, J. Rickman, C. Thomas, F. Nédélec, and T. Surrey, Determinants of polar versus nematic organization in networks of dynamic microtubules and mitotic motors, Cell 175, 796 (2018).

[49] D. Oriola, F. Jülicher, and J. Brugués, Active forces shape the metaphase spindle through a mechanical instability, Proc. Natl. Acad. Sci. USA 117, 16154 (2020).

[50] D. P. Holmes, Elasticity and stability of shape-shifting structures, Curr. Opin. Colloid Interface Sci. 40, 118 (2019). 